\title{
Polimiopatia hipocalêmica e hipertensão secundária a hiperaldosteronismo primário felino*
}

\section{Hypokalemic polymyopathy and secondary hypertension to feline primary hyperaldosteronism}

\author{
Natalia Sciammarella Razal, ${ }^{* *}$ Patrícia Veronica Ferreira Hormazabal Rodriguez, ${ }^{* *}$ \\ Mário dos Santos Filho*** Rosemar de Almeida Freitas**
}

\begin{abstract}
Resumo
O hiperaldosteronismo se define pela hipersecreção de aldosterona pelas suprarrenais, resultando em excesso de sódio e redução de potássio sanguíneo. Esta hipersecreção deve-se à síntese autônoma de aldosterona por células adrenais hiperplásicas ou neoplásicas, que agem independentemente da estimulação pelo sistema renina-angiotensina. A doença acomete felinos de adultos maduros a idosos. O excesso de aldosterona culmina em hipertensão sistêmica e/ou hipocalemia, que levam à fraqueza muscular e alterações oculares. O diagnóstico é baseado em exames laboratoriais e de imagem, e o tratamento pode ser clínico ou cirúrgico. O prognóstico é considerado favorável quando as medicações são capazes de melhorar as manifestações clínicas ou quando é possível realizar o procedimento cirúrgico. O presente trabalho visa relatar o caso de um felino macho de 13 anos, castrado, sem raça definida, com hipocalemia persistente secundária a um presuntivo tumor adrenal.
\end{abstract}

Palavras-chave: Gato, glândula adrenal, doença renal, hipocalemia, polimiopatia, Síndrome de Conn.

\begin{abstract}
Hyperaldosteronism is defined by the hypersecretion of aldosterone by the adrenal glands resulting in excess sodium and reduced blood potassium. This hypersecretion is due to the autonomous synthesis of aldosterone by hyperplastic or neoplastic adrenal cells, which act independently of stimulation by the renin-angiotensin system. The disease affects felines in the age group from mature adults to the elderly. The excess of aldosterone culminates in systemic hypertension and/or hypokalemia, which leads to muscle weakness and ocular changes. The diagnosis is based on laboratory and imaging tests and treatment can be clinical or surgical. The prognosis is considered favorable when the medications are able to improve the clinical manifestations or when it is possible to perform the surgical procedure. The present paper aims to report the case of a 13-year-old male cat, castrated, crossbred, with persistent hypokalemia secondary to a presumptive adrenal tumor.
\end{abstract}

Keywords: Cat, adrenal gland, kidney disease, hypokalemia, polymyopathy, Conn's syndrome.

\section{Introdução}

O hiperaldosteronismo é uma endocrinopatia resultante do excesso de produção de aldosterona pelas glândulas adrenais, podendo este ser primário ou secundário (Harvey; Refsal, 2012). Quando secundário, é resultante da ativação do sistema renina-angiotensina-aldosterona, em resposta a uma alteração orgânica, como nos casos de desidratação, hipotensão, hipovolemia ou déficit de sódio. No hiperaldosteronismo secundário, normalmente está associado à existência de insuficiência cardíaca congestiva, doença renal ou doença hepática, as quais potencialmente podem desencadear a ativação do sistema renina-angiotensina-aldosterona (AHS et al., 2005; Reusch et al., 2010; Galac et al., 2016; DjajadiningratLaanen et al., 2011).
Nesse caso, as concentrações de renina e angiotensina encontram-se elevadas (Galac et al., 2010). O aumento excessivo de aldosterona na circulação eleva a excreção renal de potássio, causando hipocalemia, e aumenta a reabsorção renal de sódio e água, provocando aumento de volume circulante. Concomitantemente, existe a resistência vascular periférica decorrente da ação da angiotensina, que, aliando-se à retenção de volume citada anteriormente, origina a hipertensão arterial sistêmica (Bento et al., 2016).

Já no hiperaldosteronismo primário, o excesso de aldosterona é decorrente da presença de neoplasia adrenal unilateral ou de hiperplasia bilateral. Nos felinos, a causa mais frequente desta doença são as neoplasias adrenocorticais unilaterais (Harvey \& Refsal, 2012; Combes et al., 2013).

*Recebido em 13 de março de 2020 e aceito em 15 de junho de 2020.

**Universidade Castelo Branco, Rio de Janeiro, RJ.

***Universidade Federal Rural do Rio de Janeiro.

Autor para contato: rosemar@castelobranco.br. 
Aidade média dos felinos diagnosticados com hiperaldosteronismo varia de 5 a 20 anos. Não há evidência de predisposição de raça ou de sexo (Djajadiningrat-Laanen et al., 2011). Para o diagnóstico, além de exame de imagem (ultrassonografia, radiografia e tomografia computadorizada), indica-se a dosagem hormonal de aldosterona e relação renina/aldosterona (Harvey e Refsal, 2014). O tratamento pode ser cirúrgico, em casos de afecções unilaterais, ou clínico, visando o controle dos sinais de hipertensão e hipocalemia (Galac et al., 2016).

O prognóstico é variável. Para os felinos não tratados, a média de sobrevida é de 1.5 a 4 meses. Já para os felinos submetidos ao tratamento clínico, a média é de 2.5 a 32 meses (Smith, Mayhew \& Berent, 2012). Para os felinos submetidos à adrenalectomia e sem complicações no pós-cirúrgico, a média é de 12 a 20 meses (Harvey \& Refsal, 2012; Smith et al., 2012).

O objetivo deste trabalho é relatar o caso de um felino, macho, de 13 anos, com fraqueza muscular generalizada e hipertensão secundária ao hiperaldosteronismo.

\section{Relato de caso}

Um felino macho, castrado, de 13 anos e sem raça definida, foi encaminhado ao atendimento nefrológico em uma clínica veterinária localizada na zona norte do Rio de Janeiro. Ele já vinha sendo tratado para doença renal crônica há seis meses com fluidoterapia $(150 \mathrm{~mL}$ de solução de ringer lactato, por via subcutânea, a cada 48 horas) e ração terapêutica para nefropatas. Há dois meses, recebia também tratamento para hipocalemia com citrato de potássio por via oral $(50 \mathrm{mg} / \mathrm{kg} /$ sid).

Duas semanas antes da consulta nefrológica, o paciente ficou internado por apresentar prostração, fraqueza muscular, dificuldade de apreender o alimento (disfagia), emagrecimento progressivo, desidratação (9\%), dificuldade de locomoção, andar deambulante e postura plantígrada. $\mathrm{Na}$ ocasião, o paciente apresentava as seguintes alterações no exame de sangue que havia sido realizado durante o período de internação: creatinina de $4,3 \mathrm{mg} / \mathrm{dL}$ (Ref.: 0,6-1,6mg/dL) e ureia de 185,5mg/dL (Ref.: $10-56 \mathrm{mg} / \mathrm{dL})$.

O exame ultrassonográfico da região abdominal revelou rim direito com dimensões aumentadas e o esquerdo com dimensões reduzidas, perda de relação corticomedular e aumento de ecogenicidade cortical sugerindo nefropatia. As adrenais não foram visualizadas (Figura 1).

Após alta clínica, foram mantidas a suplementação com o citrato de potássio e a fluidoterapia diária por cinco dias, acrescida de uma ampola de $10 \mathrm{mLs}$ de cloreto de potássio 19,1\%. A repetição dos exames de sangue revelou: Creatinina $=2,5 \mathrm{mg} / \mathrm{dL}$, ureia $=56,0 \mathrm{mg} / \mathrm{dl}$ e potássio $=2,7 \mathrm{mEq} / \mathrm{L}$ (Ref.: 3,7-5,8mEq/L). Na revisão seguinte, o paciente apresenta-se hipertenso $(200 \mathrm{mmHg})$, e após redução da fluidoterapia para a cada 48 horas, o paciente voltou a ter os mesmos sinais clínicos, porém, com maior intensidade e sem conseguir erguer a cabeça (Figura 2).

Foi, então, solicitada a avaliação cardiológica em virtude da presença da doença renal crônica e novos exames, já que o paciente não respondia à terapia conservadora e convencional de reposição de potássio.

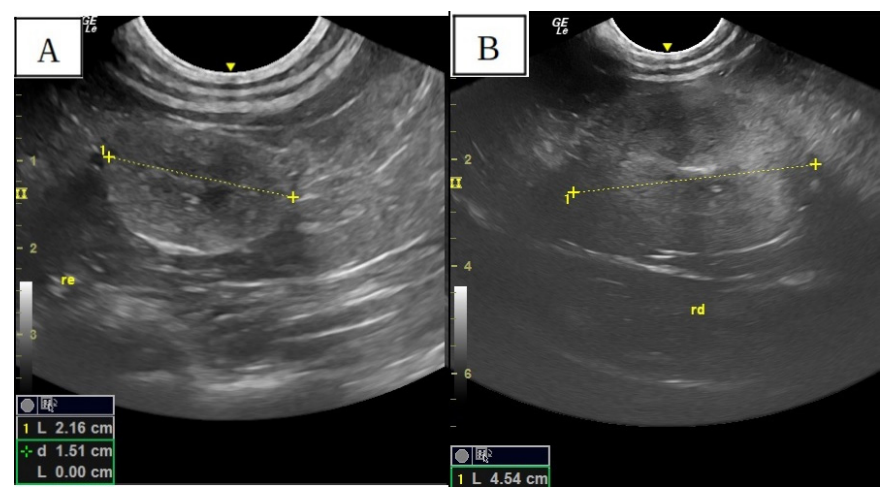

Figura 1: Imagem ultrassonográfica de um felino, macho, SRD de 13 anos, demonstrando: (A) Rim esquerdo com dimensões reduzidas, contorno regular e perda da relação corticomedular. (B) Rim direito apresentando dimensões aumentadas, perda da relação corticomedular e evidente aumento de ecogenicidade cortical.

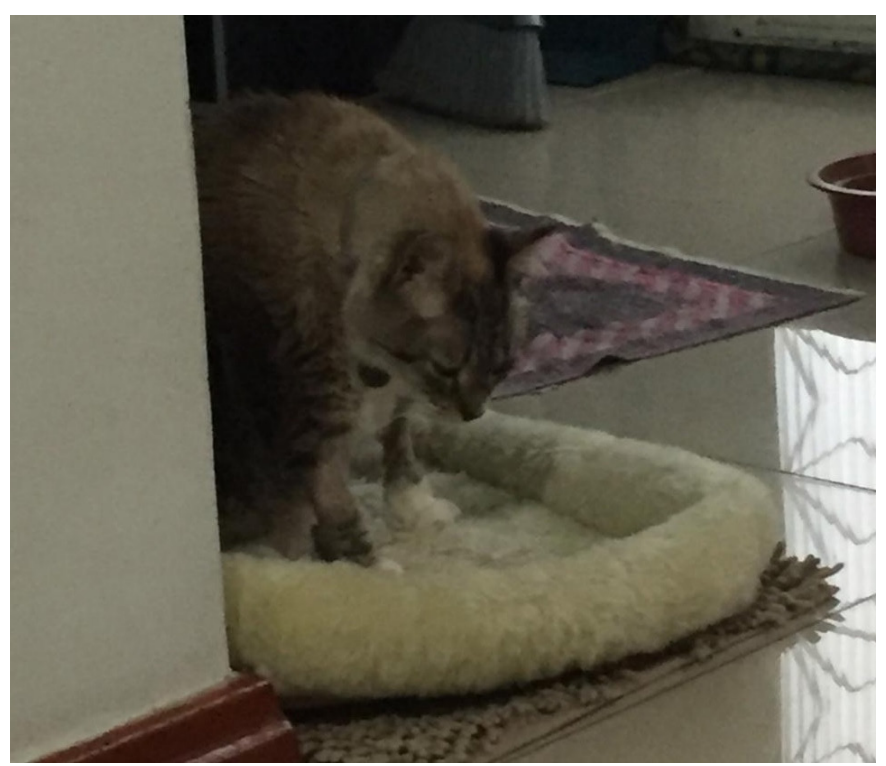

Figura 2: Felino, 13 anos. Ventroflexão cervical como consequência da hipocalemia. Ressalta-se pelame opaco e eriçado.

Em virtude da perda intensa de massa magra e da fraqueza muscular não compatíveis com o estágio da doença renal, nova amostra de sangue foi coletada para dosagem de creatinina, ureia, sódio, potássio, creatininoquinase $(\mathrm{CK})$ séricos, hemograma e dosagem hormonal de aldosterona pela técnica de radioimunoensaio. Obtiveram-se os seguintes resultados: CK 3.300,0 UI/L (Ref.: 65,0-320,0 UI/L), creatinina 2,4 mg/dL, potássio 3,1mEq (Ref.: 3,7-5,8mEq), sódio $161 \mathrm{mEq}$ (Ref.: 141,0$153,0 \mathrm{mEq}$ ) e ureia $60,4 \mathrm{mg} / \mathrm{dL}$. O hemograma não demonstrou alterações dignas de nota.

Enquanto se aguardava o resultado da dosagem hormonal de aldosterona, e diante da piora clínica do paciente, foi iniciado o tratamento para hipertensão arterial sistêmica com espironolactona $(2 \mathrm{mg} / \mathrm{kg} / \mathrm{bid})$ e besilato de anlodipino $(0,125 \mathrm{mg} / \mathrm{gato} / \mathrm{sid})$, mantendo-se a fluidoterapia a cada 48 horas. Após 48 horas do uso destas medicações, a tutora relatou que o paciente já conseguia se alimentar sozinho, erguer a cabeça e correr pela casa como costumava fazer antes do diagnóstico da doença renal; e a pressão arterial já havia 
normalizado (140mmHg). Foi então suspensa a fluidoterapia e mantida a espironolactona e o anlodipino sem a necessidade de suplementação de potássio.

Aproximadamente 15 dias após o início do tratamento, a tutora realizou o exame de ecocardiograma anteriormente solicitado e novo exame de sangue, cujos resultados estão a seguir: CK $=85,2 \mathrm{UI} / \mathrm{L}$, creatinina $=1,8 \mathrm{mg} / \mathrm{dL}$, fósforo $=4,6 \mathrm{mg} / \mathrm{dL}$ (Ref.: 2,9-7,0 mg/dL), potássio $=5,1 \mathrm{mEq} / \mathrm{L}$, sódio $=153,0 \mathrm{mEq} / \mathrm{L}$, ureia $=80,6 \mathrm{mg} / \mathrm{dL}$. O ecocardiograma não apresentou alterações dignas de nota. Neste mesmo momento foram recebidos os resultados da dosagem aldosterona e a suspeita clínica de hiperaldosteronismo foi confirmada (Aldosterona $=711,38 \mathrm{pg} /$ $\mathrm{mL}$ - Ref.: 3,3-261,3 pg/ml).

Um ano após o diagnóstico de hiperaldosteronismo, o paciente seguia clinicamente bem, ganhando peso, sem fluidoterapia, mantendo o tratamento com espironolactona e besilato de anlodipino (Figura 3).

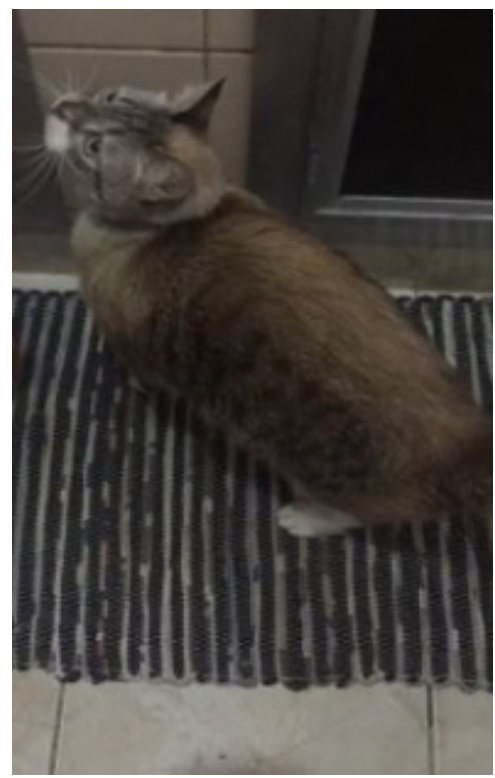

Figura 3: Imagem do paciente felino, ilustrando a melhora clínica. Ressalta-se o ganho de peso e a ausência de ventroflexão do pescoço e a melhora no pelame.

Um ano e dois meses após diagnóstico, o paciente retornou à clínica desidratado, prostrado e anorético. Novos exames de sangue e de ultrassonografia abdominal foram realizados e, nesta ocasião, foi constatada a presença de provável tumoração na glândula adrenal direita, com aspecto heterogêneo, hipoecóico e medindo aproximadamente $4,2 \times 3,11 \mathrm{~cm}$ de comprimento (Figura 4).

O paciente veio a óbito 72 horas após o retorno dos sinais clínicos. Nesta ocasião, a creatinina estava em $8,0 \mathrm{mg} / \mathrm{dL}$, a ureia em $282,9 \mathrm{mg} / \mathrm{dL}$, o sódio em $126,0 \mathrm{mEq} / \mathrm{L}$, o potássio em $2,6 \mathrm{mEq} / \mathrm{L}$, a CK em 450,6 UI/L, o cálcio iônico em 7,3 mg/dL (Ref.: 3,1 a $5,1 \mathrm{mg} / \mathrm{dL}$ ) e o cálcio total em $12 \mathrm{mg} / \mathrm{dL}$ (Ref.: 6,2 a 10,2mg/dL). Não houve tempo hábil para a repetição da dosagem hormonal de aldosterona. A responsável não autorizou a realização de necrópsia para análise histopatológica da massa adrenal.

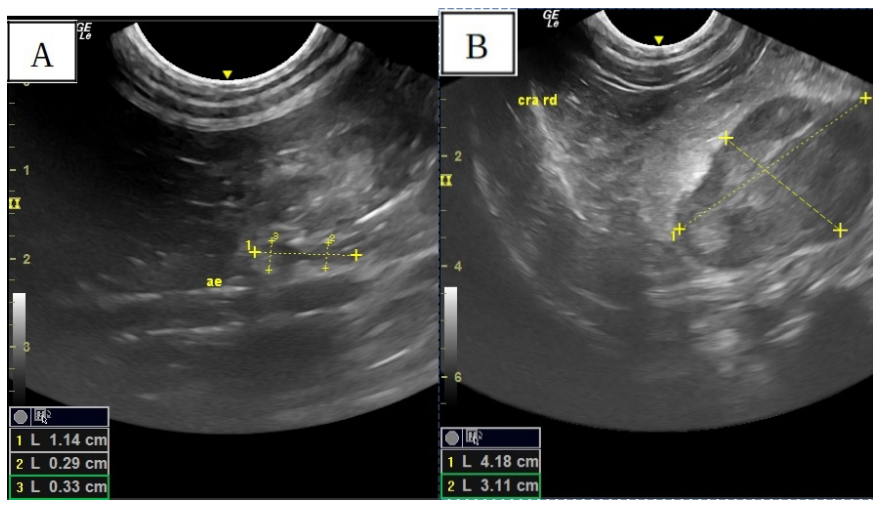

Figura 4: Imagem ultrassonográfica da glândula adrenal 1 ano e dois meses após o diagnóstico de hiperaldosteronismo. (A) Adrenal esquerda medindo $0,23 \times 1,14 \times 0,33 \mathrm{~cm}$ de comprimento, e apresentando formato anatômico, ecotextura e ecogenicidade aparentemente preservadas. (B) Adrenal direita de tamanho aumentado, medindo em torno de $4,2 \times 3,11 \mathrm{~cm}$ de comprimento, com aspecto heterogêneo e hipoecóica, sugerindo a presença de neoplasia.

\section{Discussão}

O hiperaldosteronismo é uma endocrinopatia importante na clínica de felinos, porém subdiagnosticada (Schulman, 2010). Sua ocorrência é maior entre gatos adultos ou idosos, com faixa etária média de 13 anos de idade, tal como o felino do presente relato.

A elevação da concentração sérica de creatinina observada no paciente tem relação direta com a redução da taxa de filtração glomerular ( $\geq 75 \%)$, que cursa, consequentemente, na sua retenção no organismo. No entanto, seus valores devem ser interpretados com cautela (Cobrin et al., 2013), pois a desidratação e as alterações na massa muscular influenciam a sua concentração sérica, devendo ser consideradas na interpretação do resultado, principalmente quando se trata de um paciente idoso (Nabity et al., 2015; Cannon, 2016).

Já quanto à elevação da ureia, mesmo sabendo que ela é o marcador menos confiável para o diagnóstico da doença renal em virtude de sofrer influências extra renais, como, por exemplo, uso de dieta hiperproteica, a elevação no caso relatado pode ser atribuída também à redução da taxa de filtração glomerular (Backlund et al., 2011; Cannon, 2016).

Mesmo sabendo que a hipocalemia é uma alteração comum em gatos com doença renal crônica em estágio inicial (estágio 2), mesmo estágio do paciente, já que esta cursa com um aumento do fluxo tubular e consequente redução na sua absorção renal, quando a suplementação é realizada, esse problema é solucionado (Reynolds; Lefebvre, 2013). No entanto, o que chamou a atenção neste paciente foi que ele estava recebendo suplementação oral há dois meses e ainda assim o potássio permanecia em declínio, resultando em piora clínica progressiva, mostrando que essa hipocalemia não era decorrente da DRC e justificando mais investigação acerca da ocorrência desta.

Não se levou em consideração a reposição que o paciente fazia por meio da fluidoterapia subcutânea, pois, segundo Polzin (2012), a administração de fluidos com potássio pode agravar a hipocaliemia, uma vez que a expansão do volume intravascular pode levar a um aumento da taxa de filtração glomerular e, consequentemente, da excreção renal de potássio. 
Não é incomum casos de hipertensão e hipocalemia serem atribuídos à doença renal crônica, no entanto, o hiperaldosteronismo primário deve ser investigado, uma vez que pode ocasionar ambas e acelerar a progressão da doença renal crônica (Javadi et al., 2005).

A elevação do cálcio total e ionizado no estágio final da doença, pouco antes do óbito do paciente, se deve ao importante papel dos rins na homeostase do cálcio e do fósforo. Ainda que neste paciente se tenha dosado o fósforo nessa ocasião, há evidências na literatura de que desde os estágios iniciais da DRC é possível que haja hiperfosfatemia e hipocalcemia iônica discretas que são suficientes para estimular a ativação da glândula paratiroide. A glândula, por sua vez, libera o paratormônio, com a função básica de manter a homeostase do cálcio, agindo diretamente nos ossos e nos rins e indiretamente no intestino para aumentar o cálcio sérico (Reynolds; Lefebvre, 2013).

A hipertensão é considerada uma manifestação clínica clássica do hiperaldosteronismo, pois a aldosterona estimula a reabsorção ativa de sódio e água nos túbulos renais distais, aumentando, assim, o volume vascular e a excreção urinária passiva de potássio, justificando a presença de tais manifestações encontradas (Harvey e Refsal, 2014; Djajadiningrat-Laanen et al, 2011). A presença da hipertensão neste paciente não foi nenhuma surpresa, já que este é um sinal clínico extremamente frequente nos felinos com hiperaldosteronismo (DjajadiningratLaanen et al., 2011), como demonstrado em estudo realizado por Reusch e colaboradores (2010) com 30 felinos portadores de hiperaldosteronismo primário, onde 26 (86,7\%) felinos apresentavam hipertensão.

Inicialmente acreditava-se que essa hipertensão era consequência do aumento do volume vascular, induzido pela reabsorção tubular renal de sódio e água e mediado pela aldosterona. Entretanto, essa teoria não fazia sentido, uma vez que essa hipertensão contínua levaria à ativação de mecanismos compensatórios, os quais restituiriam a homeostasia vascular, regularizando, assim, a pressão arterial (Tomaschitz et al., 2010). Alguns estudos realizados nos últimos anos descrevem que este não é o único papel da aldosterona, pois esta tem forte influência não só na regulação do tônus vascular, como também na estimulação simpática. Atualmente já se sabe que esta hipertensão sistêmica secundária ao hiperaldosteronismo primário é consequência de um conjunto de eventos, tais como o aumento da reabsorção tubular renal de sódio e água, com consequente aumento do volume intravascular; associado à estimulação simpática, à indução de vasoconstrição e ao aumento da resistência vascular periférica (Reusch et al., 2010; Tomaschitz et al., 2010; Jepson, 2011).

Sabendo que os rins são considerados órgãos alvos da hipertensão não se pode descartar a possibilidade desta influenciar no valor da creatinina sérica do paciente, já que antes de iniciar o tratamento para hipertensão $(200 \mathrm{mmHg})$, e o fato de que a creatinina era de $2,5 \mathrm{mg} / \mathrm{dL}$ e após 48 horas de tratamento houve não só redução da pressão $(140 \mathrm{mmHg})$ como também da creatinina $(1,8 \mathrm{mg} / \mathrm{dL})$. Isso pode ser explicado pelo fato de que a hipertensão sobrecarrega os mecanismos de autorregulação renal, levando ao aparecimento de glomerulosclerose, destruição da barreira glomerular, liberação de mediadores e citocinas próinflamatórios, estimulando a progressão da doença renal. Além disso, a aldosterona também induz a oxidação das células tubulares renais, contruindo mais ainda para esta progressão (Jepson, 2011; Queisser et al., 2011). Contudo, mais estudos são necessários para confirmar essa hipótese (Jepson, 2011; Galac et al, 2016; Jiménez, 2012).

A hipertrofia do ventrículo esquerdo, que é relatada em $74-85 \%$ dos felinos hipertensos, não foi observada neste paciente, mesmo este estando dentro da população de felinos geriatras (Jepson, 2011).

A fraqueza muscular, disfagia e postura plantígrada observadas no paciente se correlacionam com a hipocalemia, pois é sabido que o potássio é um íon fundamental para o funcionamento do sistema neuromuscular por interferir no potencial de repouso das células, influenciado pelos níveis de potássio intracelular e extracelular (Gandolfi et al., 2012).

Em condições fisiológicas, 98\% do potássio encontra-se localizado no interior das células, e, quando há uma redução da sua concentração no meio extracelular, estas células cedem o potássio para o meio extracelular na tentativa de manter a homeostase. Quando esse potássio é cedido para o meio extracelular, em situação de hipocaliemia, isso acarreta em aumento do potencial de repouso da célula, ou seja, uma hiperpolarização, que torna a célula menos sensível aos estímulos, surgindo, assim, essa fraqueza muscular, ou, como também é chamada, polimiopatia hipocaliêmica. No entanto, essa alteração neuromuscular é observada quando a concentração de potássio extracelular se aproxima de $2,5 \mathrm{mEq} / \mathrm{L}$, e, no paciente em questão, no momento desta manifestação clínica, a concentração de potássio era de $2,7 \mathrm{mEq} / \mathrm{L}$, corroborando com o descrito na literatura (Galac et al., 2010; Feldman; Churc, 2010; Bento, 2016).

O aumento da enzima creatinoquinase (CK) pode ser justificado pela miopatia causada pela hipocalemia, já que esta é uma enzima citosólica muscular liberada para o exterior quando os miócitos são danificados (Barger, 2015).

O diagnóstico de hiperaldosteronismo, inicialmente, foi baseado na elevação dos níveis séricos de aldosterona plasmática realizada pelo método de raioimunoensaio, em associação com as alterações laboratoriais (sódio e potássio), já que a visualização da massa em adrenal direita, por meio do exame ultrassonográfico, só foi possível um ano após o diagnóstico. Até então, ambas as adrenais permaneciam inalteradas (Djajadiningrat-Laanen et al, 2011). No entanto, ainda que fosse visualizada desde o início, esta, sozinha, não seria suficiente para um diagnóstico definitivo de hiperaldosteronismo, já que outras alterações como o hiperadrenocorticismo, feocromocitomas, tumores não funcionais e tumores secretores de progesterona produzem a mesma imagem ultrassonográfica, porém, auxilia a diferenciar a forma primária da secundária da doença (Declue et al., 2005).

O tratamento do hiperaldosteronismo é baseado em drogas para controle clínico da hipertensão e hipocalemia, ou, idealmente, na retirada cirúrgica da adrenal afetada. As drogas indicadas no tratamento clínico incluem bloqueador dos canais de cálcio (anlodipino), diurético poupador de potássio (espironolactona) e a reposição de potássio (gluconato ou citrato de potássio) (Harvey; Refsal, 2014).

No presente trabalho foram utilizadas todas as drogas recomendadas pela literatura, pois estas promoveram 
melhora clínica expressiva do paciente. A espironolactona é um antagonista dos receptores de aldosterona, que inibem a reabsorção de sódio no tubo contornado distal do rim e no ducto coletor. A escolha da amlodipina é atribuída a seu efeito bloqueador dos canais de cálcio e, dessa forma, reduzindo a póscarga, e, consequentemente, o trabalho cardíaco. Além disso, ela propicia o aumento do intervalo diastólico, resultando em maior irrigação coronariana e, como resultado, uma maior nutrição e oxigenação do miocárdio (Stepien, 2011). Essa associação de drogas foi capaz de manter uma boa qualidade de vida para o paciente por um ano, ou seja, durante todo o período em que a massa não era visível.

Não houve tempo hábil para realização da adrenalectomia, pois, logo após o aparecimento da massa, o paciente foi a óbito. Vale ressaltar que a remoção cirúrgica da glândula é o tratamento de escolha para hiperaldosteronismo primário, pois apresenta a vantagem de remissão completa da hipocalemia

\section{Referências}

ASH,R.A.; HARVEY, A.M.; TASKER, S. Primary hyperaldosteronism in the cat: a series of 13 cases. Journal of Feline Medicine and Surgery. v.7, n.3, p. 173-182, 2005.

BARGER, A.M. Clinical chemistry. In: BARGER, A.M. \& MACNEILL, A.L. (Eds), Clinical Pathology and Laboratory Techniques for Veterinary Technicians. Willey Blackwell, 2015. p.95-139.

BENTO, D.D.; ZAHN, F.S.; DUARTE, L.C.; MACHADO, L.H.A. Feline primary hyperaldosteronism: an emerging endocrine disease. Ciência Rural. v. 46, n.4, p.686-693, 2016.

BACKLUND, B.; ZORAN, D.L.; NABITY, M.B.; NORBY, Bo.; BAUER, J.E. et al. Effects of dietary protein content on renal parameters in normal cats. Journal of Feline Medicine and Surgery. v. 13, n.10, p.698-704, 2011.

CANNON, M. Diagnosis and investigation of chronic kidney disease in cats. In Practice. v. 38, n. 3, p.2-9, 2016.

COBRIN, A.R.; BLOIS, S.L.; KRUTH, S.A.; ABRAMS-OGG, A.G.G.; DEWEY, C. Biomarkers in the assessment of acute and chronic kidney diseases in the dog and cat. Journal of Small Animal Practice, v. 12, n. 54, p.647-655, 2013.

COMBES, A.; PEY, P.; PAEPE, D.; ROSENBERG, D.; DAMINET, S.; PUTCUYPS, I.; BEDU, A.S.; DUCHATEAU, L.;FORNELTHIBAUD, P.; BENCHEKROUN, G.; SAUNDERS, J.H. Ultrasonographic appearance of adrenal glands in healthy and sick cats. Journal of Feline Medicine and Surgery. v. 15, n. 6 , p.445-457, 2013.

DEClUE, A. E.; BRESHEARS L. A.; PARDO, I. D.; KERL, M. E.; PERLIS, J.; COHN, L. A.Hyperaldosteronism and hyperprogesteronism in a cat with an adrenal cortical carcinoma. Journal of Veterinary Internal Medicine, v. 19, n. 1, p.355-358, 2005.

DJAJADININGRAT-LAANEN, S.; GALAC, S.; KOOISTRA, $\mathrm{H}$. Primary hyperaldosteronism: expanding the diagnostic net. Journal of Feline Medicine and Surgery. v. 13, n. 9, p. 641-650, 2011.

FELDMAN, E.C; CHURC, D.B. Electrolyte disorders: potassium. In: ETTINGER, S.J. \& FELDMAN, E,C. Textbook of Veterinary Internal Medicine. 7ed. St. Louis: Elsevier, 2010. p.303-307. e da hipertensão (Galac et al., 2010). No entanto, há risco de complicações como hemorragias, desequilíbrio hidroeletrólito, hipotensão, arritmias, tromboembolismo pulmonar e coagulação intravascular disseminada (Fossum, 2013).

As principais neoplasias mais frequentes de adrenal são os adenomas e os adenocarcinomas e, por isso, o exame histopatológico é essencial para essa diferenciação (Djajadiningrat-Laanen et al., 2011), no entanto, não foi possível de ser realizado, pois não foi autorizado pela tutora.

\section{Conclusão}

O hiperaldosteronismo é uma doença endócrina, cujos principais sinais clínicos são resultados da hipocalemia e da hipertensão arterial. Dessa forma, se faz necessário uma atenção maior diante de um felino com tais sinais bem como uma investigação se estes são consequência ou não da doença renal crônica.

FOSSUM, T.W. Adrenalectomy. In: FOSSUM, T.W. Small Animal Surgery. 4ed. Philadelphia: Mosby Elsevier, 2013. p.633-637.

GALAC, S. Adrenals. In: RIJNBERK, Ad.; KOOISTRA, H.S. (eds.) Clinical endocrinology of dogs and cats: an illustrated text. $2^{\mathrm{a}}$ ed.. Hannover: Schlutersch, 2010, p.93-140.

GANDOLFI, B.; GRUFFYDD-JONES, T.J.; MALIK, R.; CORTES, A.; JONES, B.R.; HELPS, C.R., PRINZENBERG, E.M., ERHARDT, G.; LYONS, L.A. First WNK4-hypokalemia animal model identified by genome-wide association in burmese cats. Public Library of Science. v. 7, n.1, p.1-9, 2012.

GOTO, A.; TAKAHASHI, Y.; KISHIMOTO, M.; MINOWADA, S.; AIBE, H.; KAJIO, H.; NODA, M. Primary aldosteronism associated with severe rhabdomyolysis due to profound hypokalemia. Internal Medicine. v. 48, n. 4, p. 219-223, 2009.

HARVEY, A.M; REFSAL, K.R. Primary Hyperaldosteronism. In: HARVEY, A.M; REFSAL, K.R (eds.) Clinical Endocrinology of Companion Animals, $1^{\mathrm{a}}$ ed. USA: Elsevier, 2014, p.115-127.

JAVADI, S.; DJAJADININGRAT-LAANEN, S. C.; KOOISTRA, H. S.; VAN DONGEN, A.M.; VOORHOUT, G.; VAN SLUIJS, F.J.; VAN DENINGH, T.S.G.A.M.; BOER, W.H.; RIJNBERK, A. Primary hyperaldosteronism, a mediator of progressive renal disease in cats. Domestic Animal Endocrinology, v. 28, n. 1, p.85-104, 2005.

JEPSON, R.E. Feline systemic hypertension: classification and pathogenesis. Journal of Feline Medicine and Surgery. v.13, n. 1, p.25-34, 2011.

JIMÉNEZ, B.M. Hiperaldosteronismo Primario en gatos. ¿Una endocrinopatía emergente? Revista centro veterinário, v. 53, n. 1 , p.12-20, 2012.

NABITY, M.B.; LEES, G.E.; BOGGESS, M.M; YERRAMILLI, M.; OBARE, E.; YERRAMILLI, M.; RAKITIN, A.; AGUIAR, J.; RELFORD, R. Symmetric dimethylarginine assay validation, stability, and evaluation as a marker for the early detection of chronic kidney disease in dogs. Journal of Veterinary Internal Medicine, v. 4, n. 29, p.1036-1044, 2015.

POLZIN, D.J. Chronic Kidney Disease: hypokalemia and potassium depletion. In: ETTINGER, S.J. \& FELDMAN, E.C. Textbook of Veterinary Internal Medicine. 7ed. St. Louis: Elsevier, 2012. p.2002-2003. 
QUEISSER, N.; SCHUPP, N.; STOPPER, H.; SCHINZEL, R.; OTEIZA, P.I. Aldosterone increases kidney tubule cell oxidants through calcium-mediated activation of NADPH oxidase and nitric oxide synthase. Free Radical Biologyand Medicine, v. 51, n. 11, p.1996-2006, 2011.

REUSCH, C.E.; SCHELLENBERG, S.; WENGER, M. Endocrine hypertension in small animals. Veterinary Clinics of North America: Small Animal Practice, v. 40, n. 2, p.335-352, 2010.

REYNOLDS, B.S; LEFEBVRE, H.P. Feline CKD: Pathophysiology and risk factors--"What do we know? Journal of Feline Medicine and Surgery, v. 15, Suppl. 1, p.3-14, 2013.
ROSE, S.A.; KYLES, A.E.; LABELLE, P.; PYPENDOP, B.H.; MATTU, J.S.; FOREMAN, O.; RODRIGUEZ, C.O.; NELSON, R.W. Adrenalectomy and caval thrombectomy in a cat with primary hyperaldosteronism. Journal of the American Animal Hospital Association, v. 43, n. 4, 209-214, 2007.

SCHULMAN, R.L. Feline primary hyperaldosteronism. Veterinary Clinics of North America: Small Animal Practice. v. 40, n. 2, p. 353-359, 2012.

TOMASCHITZ, A.; PILZ, S.; RITZ, E.; OBERMAYER-PIETSCH, B.; PIEBER, T.R. Aldosterone and arterial hypertension. Nature Reviews Endocrinology, v. 6, n.2, p.83-93, 2010. 